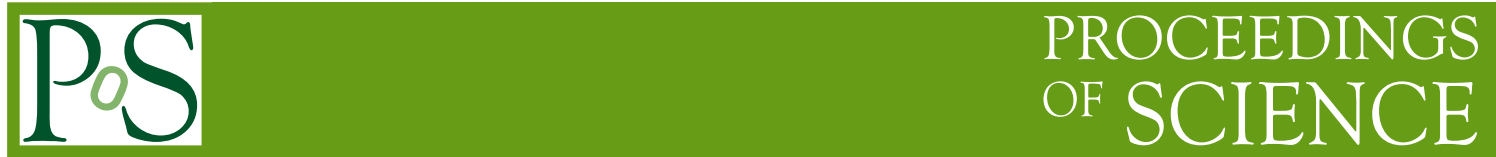

\title{
CMS Detector Global Status
}

\author{
Wesley H. Smith ${ }^{* \dagger}$ \\ University of Wisconsin - Madison \\ E-mail: wsmith@hep.wisc.edu
}

The Compact Muon Solenoid (CMS) experiment is a general purpose $p p$ detector located at the CERN Large Hadron Collider (LHC). During 2008 and 2009 the CMS detector was successfully commissioned with sustained periods of running with cosmic rays, secondary particles from LHC beam shots on the upstream collimators and single beam steered though the detector. The software and computing systems have been tested in analysis of these events and through exercises with large amounts of simulated data.

XXth Hadron Collider Physics Symposium

November 16-20, 2009

Evian, France

\footnotetext{
${ }^{*}$ Speaker.

${ }^{\dagger}$ On behalf of the CMS Collaboration.
} 


\section{Introduction to the CMS Detector}

The Compact Muon Solenoid (CMS) experiment is a general purpose $p p$ detector. CMS consists of a large diameter superconducting solenoid containing an all-silicon tracker, a crystal electromagnetic calorimeter (ECAL) and a brass-scintillator hadron calorimeter (HCAL), with a return yoke and muon chambers outside. The silicon tracker consists of a vertex detector with 66 million pixel channels and a silicon strip tracker with 9.6 million channels, for a total of $210 \mathrm{~m}^{2}$ of silicon sensors. The electromagnetic calorimeter consists of 76,000 scintillating $\mathrm{PbWO}_{4}$ crystals in the barrel and endcap. The hadronic calorimeter is composed of a plastic scintillator and brass sandwich. The iron core superconducting solenoid operates at a field of $3.8 \mathrm{~T}$. Outside the solenoid is a sampling scintillator hadronic outer $(\mathrm{HO})$ calorimeter used as a tail-catcher. The steel yoke of the magnet system is instrumented with muon chambers. The barrel contains Drift Tubes (DT) and Resistive Plate Chambers (RPC). The endcap contains Cathode Strip Chambers (CSC) and RPCs. Further information on the CMS detector is available in $[1,2]$.

\section{CMS Operations in 2008}

The CMS Detector was first closed for LHC beam operation on September 3, 2008. In the subsequent days CMS observed "splash" events from beam shots hitting the upstream collimator. The interactions of the beam with the collimator and surrounding material produced a large quantity of secondary particles which passed through the CMS detector, typically depositing $\sim 100 \mathrm{TeV}$ in the ECAL and $\sim 1000 \mathrm{TeV}$ in the HCAL. These events were used to internally synchronize the CMS ECAL and HCAL, as well as to inter-calibrate the ECAL channels. From September 10 - 19 single beams were circulated and beam halo events were observed in CMS. The beam halo events were used to internally synchronize and align the CMS muon systems.

During October-November 2008, after the period of beam operation, CMS began a campaign of continuous operation taking cosmic ray data to gain operational experience and to test the stability of the experimental infrastructure. Dubbed the "Cosmic Run At Four Tesla" (CRAFT08) this involved running the detector with the Solenoid at its operational field of $3.8 \mathrm{~T}$ 24/7 for six weeks. The efficiency of operation averaged $70 \%$. Approximately $300 \mathrm{M}$ cosmic events were collected and $400 \mathrm{~TB}$ of resulting data was distributed to collaborating CMS institutions world-wide. The first analyses of these data used the software release intended for 2008 data-taking and the LHC grid infrastructure. This was followed by re-reconstruction and analyses with more advanced versions of the software. The analysis produced results such as measurements of the barrel pixel and inner tracker alignment, muon energy deposits in the ECAL and HCAL and the position resolution of the muon chambers. Over 20 papers containing results from this running are being submitted to the Journal of Instrumentation (JINST).

After the conclusion of CRAFT08 in November, 2008, the CMS detector was opened for maintenance and repair activities, the installation of the pre-shower (ES) detector and CASTOR (Centauro And Strange Object Research), a quartz-tungsten sampling calorimeter[2], designed for the very forward rapidity region in heavy ion and proton-proton collisions. There were a number of major accomplishments during this shutdown. The forward pixel system was removed, repaired and re-inserted. The preshower detector was commissioned. The Tracker cooling plant was re- 
vamped. Meticulous studies of the magnetic field produced new understanding and a revised field map with agreement between Monte Carlo calculations and data measurements better than $2 \%$.

\section{CMS Cosmic Running and Computing/Software Tests in 2009}

After the conclusion of the 2008-2009 shutdown, CMS began a 40 day campaign of running with cosmic rays (CRAFT09) from July 23 - September 1, 2009. The operation was mostly continuous with a few planned downtimes. The CMS DAQ system ran efficiently at an $80 \mathrm{kHz}$ L1 trigger rate input ( $1 \mathrm{kHz}$ cosmic ray plus calibration events and $80 \mathrm{kHz}$ random triggers) with all systems in, including the preshower. The higher level trigger ran over 4700 applications on 672 filter unit PCs processing $4 \times 10^{9}$ events in $\sim 15$ hours. A sample of $300 \mathrm{M}$ cosmic events was collected with the field on at $3.8 \mathrm{~T}$ and $160 \mathrm{M}$ with the field off. The quality of the data from and the performance of CMS was equal to or better than that of CRAFT08. The data-taking efficiency was $\sim 80 \%$ on average and on occasion (e.g. weekends) was $\sim 85-90 \%$.

During CRAFT09, on average $98.5 \%$ of the silicon pixel detector and $98.1 \%$ of the silicon strip tracker were operational. To evaluate the tracking efficiency, muons were tagged by the barrel muon chambers. The two track-finding algorithms used were a combinatorial track-finder (CTF) designed for collisions and a cosmic track finder (CosmicTF) based on a simple algorithm tailored for muon tracks. The latter provides a cross check. The efficiencies were $99.8 \%$ and $99.8 \%$ for the CTF and CosmicTF respectively. The efficiency as a function of $p_{T}$ is shown in Figure 1 . The hit efficiency in various layers of the silicon tracker was also measured in CRAFT09. Tracks were searched for without consideration of the layer being investigated. The track was extrapolated into the layer being investigated and a hit searched for in the expected region. For the data sample studied, the hit efficiency was $98.2 \%$. When known faulty modules are included, the efficiency is 99.9\%. The hit efficiency for various layers of the Tracker is shown for field on and off in Figure 2.

99\% of the Endcap Muon CSC Chambers were also operating and taking data during CRAFT09. Figure 3 shows the reconstructed hits in $\mathrm{x}$ and $\mathrm{y}$ for all 8 planes of the CSC muon chambers moving outward from the detector center from left to right with the + endcap on top (ME+1 to +4$)$ and the - endcap on the bottom (ME-1 to -4). This figure is taken from one run on August 11, 2009 where $\mathrm{LV}$ power supplies had been borrowed from some of the ME+4 chambers for a test.

During CRAFT09, the CMS Level-1 (L1) trigger provided DT, CSC and RPC muon triggers and ECAL and HCAL based triggers with very low thresholds. The efficiencies were high and threshold turn-on curves were sharp in spite of the low thresholds. The efficiency of the L1 DT track-finder trigger as determined by selecting and analyzing muons in the silicon tracker that point through the CMS interaction point is shown in Figure 4 for a threshold of $10 \mathrm{GeV}$. The efficiency of the L1 $e / \gamma$ trigger was measured using the bremsstrahlung of cosmic ray muons in the ECAL crystals. The study used muon triggered events, requiring a muon associated with the ECAL electromagnetic cluster. The efficiency of the $\mathrm{L} 1 e / \gamma$ trigger for 1,5 , and $10 \mathrm{GeV}$ thresholds is shown in Figure 5.

While CMS was taking Cosmic Data in preparation for beam, the CMS software and computing systems were also preparing for collision data. Between July and October 760 Million full simulation events were generated at both 7 and $10 \mathrm{TeV}$ cms energy. These events were used in continuous tests of the readiness of the CMS Tier-1and Tier- 2 computing centers. The Tier- 1 com- 


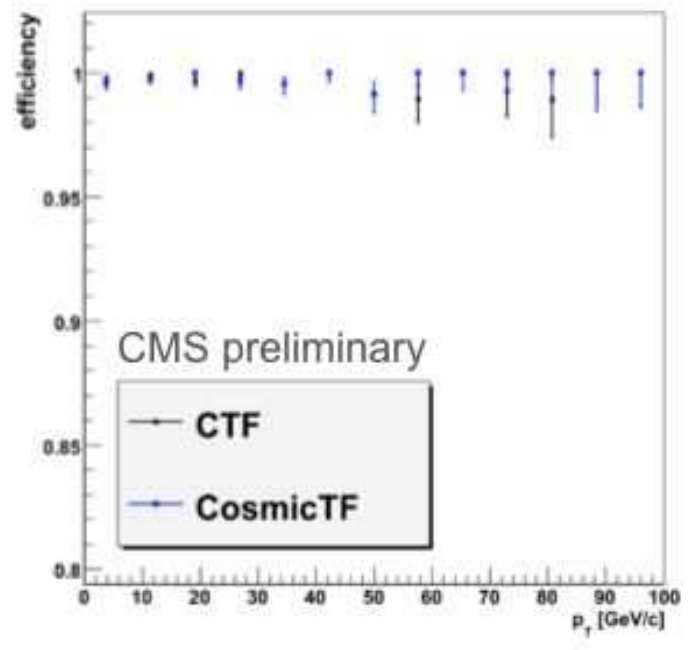

Figure 1: Track-finding efficiency of the CMS Central Tracker vs. $p_{T}$ for cosmic tracks tagged by the barrel muon chamber using the CTF and CosmicTF tracking algorithms.

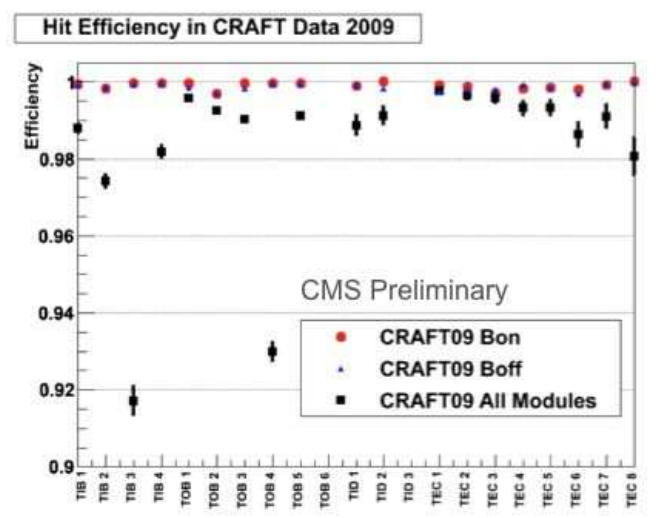

Figure 2: Hit efficiency of the silicon tracker for various layers in the Tracker Inner Barrel (TIB), Outer Barrel (TOB), Inner Disk (TID) and Endcap (TEC).
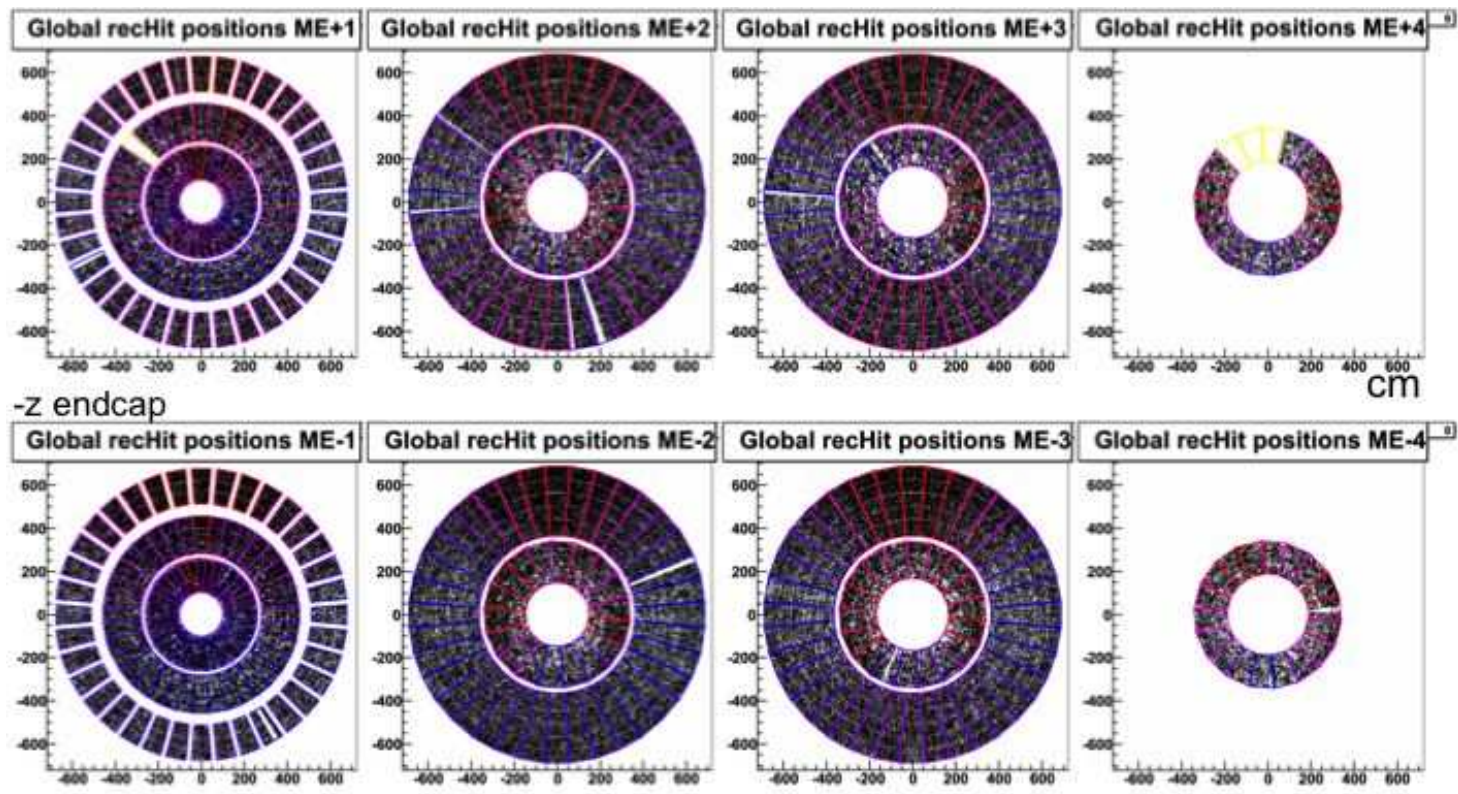

Figure 3: Reconstructed hits in the 8 planes of Endcap Muon CSCs. 


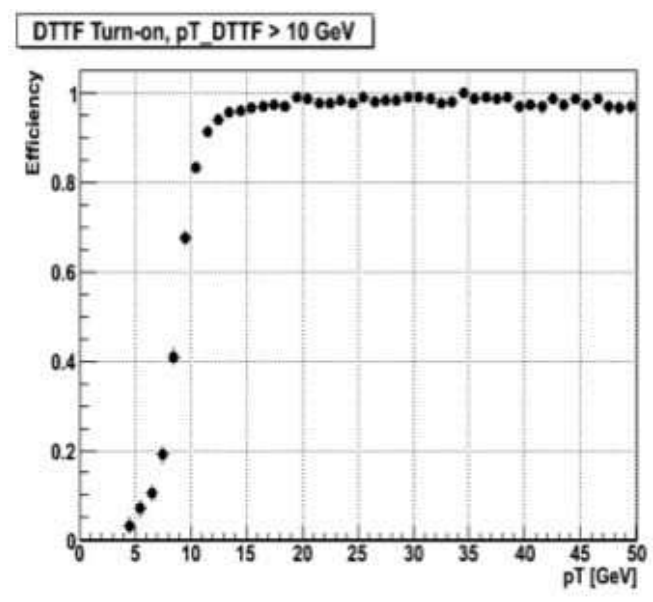

Figure 4: L1 DT track-finder trigger efficiency from muons measured in the silicon tracker pointing through the CMS interaction point.

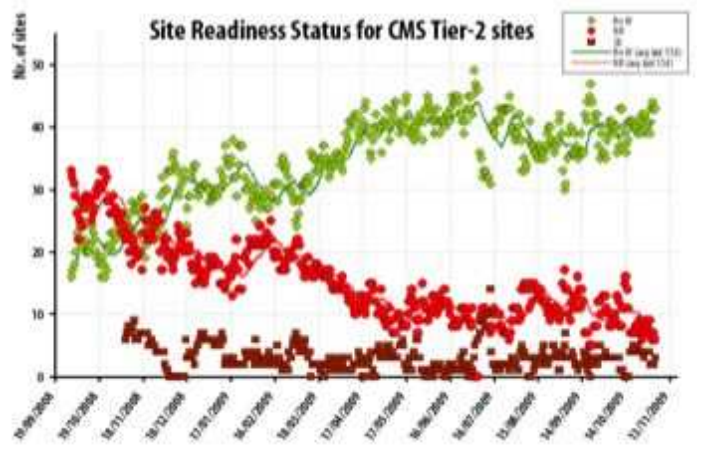

Figure 6: Status of CMS Tier-2 centers over time showing ready and working, not ready and scheduled downtime.

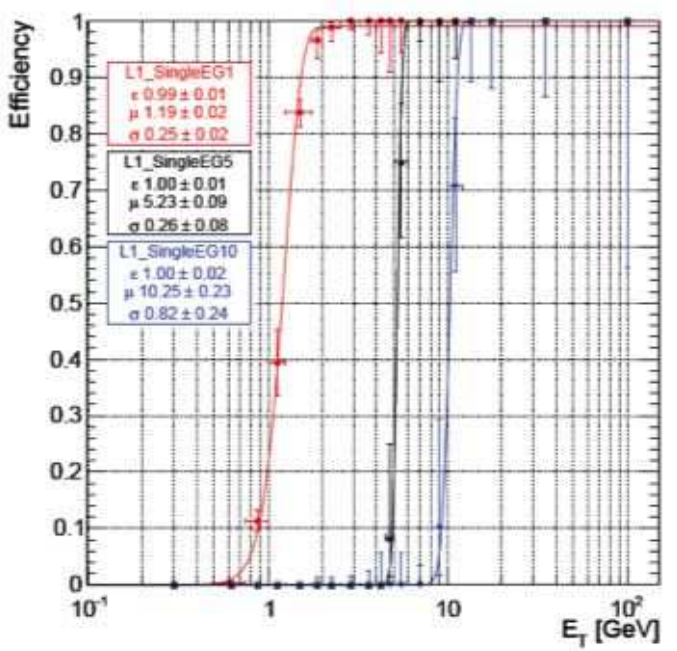

Figure 5: The efficiency of the L $1 e / \gamma$ trigger for 1,5 , and $10 \mathrm{GeV}$ thresholds measured using muon bremsstrahlung.

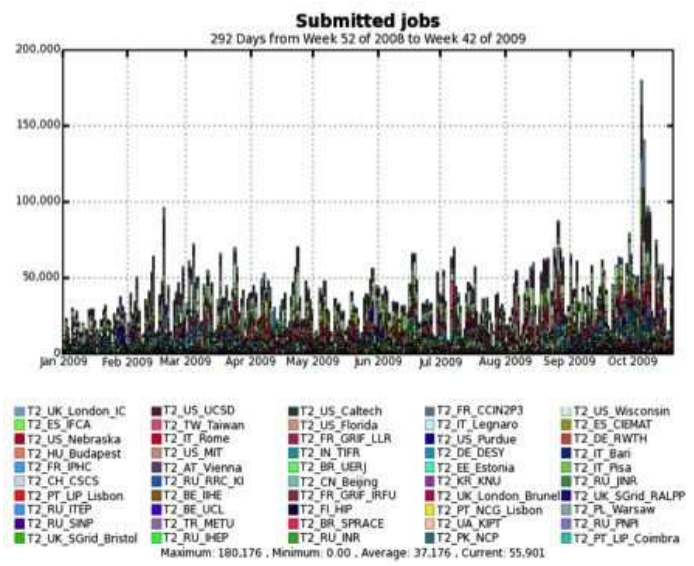

Figure 7: Submitted jobs to the CMS Tier-2 Centers per day showing the increment of the "October Exercise."

puting facilities are major repositories of $\mathrm{MC}$ and reconstructed data while the Tier-2 facilities are half devoted to simulation and half to user analysis and serve as the primary resource for analysis. The readiness of the Tier- 2 sites is closely monitored as shown in Figure 6. There was an intensive test of the CMS analysis chain software and computing during a 2-week "October Exercise" where 77 separate analyses were run with 230 participants, adding an increment of 700k jobs to the normal computing load. This increment is shown in Figure 7. During the exercise there were $\sim 2000$ data subscriptions and 900 TB moved from Tier-1's to Tier-2's. 


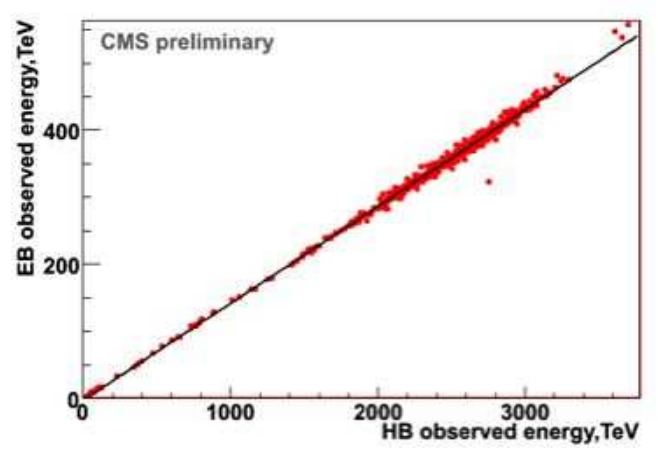

Figure 8: Barrel ECAL vs. HCAL energy observed in "splash" events.

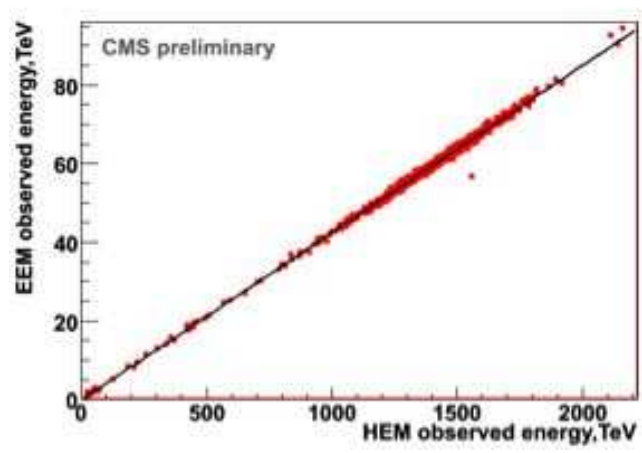

Figure 9: Minus endcap ECAL vs. HCAL energy observed in "splash" events.

\section{CMS Beam "Splash" Running in 2009}

The LHC started to deliver beam shots to the collimators upstream of CMS on November 7. CMS collected 1105 "splash" events while operating the detector with the magnetic field at 3.8 T. To ensure safety, the silicon strip and pixel tracking systems were off and the muon systems (DT, CSC, RPC) were at reduced high voltage. The calorimeters were operating and the detector was triggered by ECAL towers over threshold in the central $\eta$ ring. This was the first exposure to beam for the preshower, CASTOR, and the Zero Degree Calorimeter (ZDC), all of which were operating. 721 "splash" events were used in a study of the energy observed in the ECAL compared with that in the HCAL. This comparison is shown in Figures 8 and 9 for the ECAL and HCAL Barrel and - endcap, respectively. Note that since there are millions of muons per event, there are 1000's of $\mathrm{TeV}$ in each event. There is good correlation between the CMS HCAL and ECAL energies over this vast energy range.

\section{Conclusions}

The CMS detector has been closed after a successful 10-month maintenance period and is taking data in round-the-clock operation with the magnet at its operational field of $3.8 \mathrm{~T}$. All subdetectors are operating with good efficiency. CMS took and analyzed "beam splash" data from beam shots on the upstream collimators and presented results a few days later. The data analyzed from cosmic and "beam splash" running shows an aligned and calibrated detector with trigger and data acquisition systems ready for physics. The computing and software systems have been exercised to demonstrate readiness for data analysis.

\section{References}

[1] S. Chatrchyan et al., (CMS Collaboration), JINST, 3 (2008) S08004.

[2] CMS Collaboration, CMS Physics Technical Design Report, Volume 1: Detector Performance and Software, CERN/LHCC 2006-001; CMS Collaboration, CMS Physics Technical Design Report, Volume 2: Physics Performance, J. Phys. G: Nucl. Part. Phys., 34 (2007) 995. 